\title{
Estimating Flow Rate Using the Motor Torque in a Rotary Blood Pump
}

\author{
Wataru Hijikata*, Jun Rao ${ }^{1}$, Shodai Abe ${ }^{1}$, \\ Setsuo Takatani ${ }^{2,3}$ and Tadahiko Shinshi \\ Precision and Intelligence Laboratory, Tokyo Institute of Technology, \\ 4259 Nagatsuta-cho, Midori-ku, Yokohama 226-8503, Japan \\ 'Interdisciplinary Graduate School of Science and Engineering, Tokyo Institute of Technology, \\ 4259 Nagatsuta-cho, Midori-ku, Yokohama 226-8503, Japan \\ ${ }^{2}$ Division of Research and Development, MedTech Heart Inc., \\ 3-24-8 Asakusabashi, Taito-ku, Tokyo 111-0053, Japan \\ ${ }^{3}$ School of Medicine, Nihon University, \\ 30-1 Ooyaguchikami-cho, Itabashi-ku, Tokyo 173-8610, Japan
}

(Received July 30, 2014; accepted March 12, 2015)

Key words: flow rate estimation, viscosity estimation, rotary blood pump, motor torque

Sensorless technologies for estimating the flow rate in rotary blood pumps are desirable in that they can enable the realization of cost-effective and compact circulation support systems without the need for flow meters. A simple method for estimating the flow rate in a centrifugal blood pump using the measured torque and rotational speed of the motor is proposed and tested. By utilizing the motor torque measured using a torque meter, the error in estimating the flow rate of water was found to be less than 0.51 $\mathrm{L} / \mathrm{min}$ in the range from 0 to $10 \mathrm{~L} / \mathrm{min}$, which is about half the equivalent value using the torque estimated with the torque monitor function provided in a commercially available motor driver. Furthermore, to compensate for changes in blood viscosity, the outlet port of the pump was clamped and the viscosity was estimated using the measured torque and rotational speed of the motor. The error in estimating the viscosity was less than $0.16 \mathrm{mPa} \cdot \mathrm{s}$ in the range from 0.75 to $3.29 \mathrm{mPa} \cdot \mathrm{s}$. The estimated flow rate, compensated by the estimated viscosity, showed an error of less than $0.77 \mathrm{~L} / \mathrm{min}$ in the range up to $10 \mathrm{~L} / \mathrm{min}$. Although the proposed method for estimating flow rate is described by a simple equation and its calibration is easier, the accuracy is comparable to that of conventional methods.

"Corresponding author: e-mail: hijikata.w.aa@m.titech.ac.jp 


\section{Introduction}

According to a report by the World Health Organization, heart failure is the predominant cause of death in the world. ${ }^{(1)}$ Ventricular assist devices (VADs), which use blood pumps to support failing hearts, have been proposed as an alternative to heart transplants. Recently, rotary blood pumps (RBPs), the impellers of which are levitated using magnetic or hydrodynamic bearings and rotated by motors to generate continuous blood flow, have been developed in order to enhance their durability, decrease the damage to blood cells and avoid blood coagulation. ${ }^{(2,3)}$

In addition to the development of RBP devices, sensorless technologies for estimating the flow rate produced by the RBPs are also an area of focus. ${ }^{(4)}$ It is desirable to measure the blood flow rate to control the operating point of the RBP in order to accommodate the physiological demands from a patient's body. Moreover, there is a need to measure the flow rate in an RBP to enable the use of novel therapies that promote heart recovery, in which the flow rate of the RBP is controlled and the load to the biological heart intentionally increased. ${ }^{(5)}$ However, most implantable RBPs avoid using flow meters because of their high cost, complexity, large size and the possible

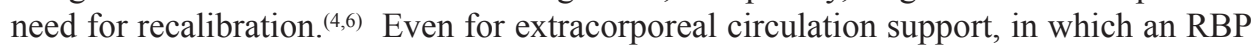
and a flow meter are placed outside the body, it is desirable to eliminate the flow meter to achieve a compact size for portable use.

An alternative method for measuring flow is to use sensorless technology, which ordinarily utilizes the motor current or motor power as well as its rotational speed. The technical challenges are (i) to simplify the equation used to estimate flow rate with sufficient accuracy and (ii) to compensate for the effect of changes in blood viscosity. Meeting the former challenge is required in order to make the calibration process easier. The latter challenge needs to be met in order to achieve accurate estimations. The motor current and power, in particular, not only depend on the flow rate but also on the blood viscosity. In addition to individual differences, the blood viscosity can change even in the same patient. For example, the volume ratio of red blood cells (hematocrit) can decrease from the normal value of $40 \%$ to less than $21 \%$ due to transfusions. ${ }^{(7)}$ In this case, the blood viscosity, which is related to the hematocrit, can be between that of plasma $(1.2 \mathrm{mPa} \cdot \mathrm{s})$ and that of normal blood (about $3 \mathrm{mPa} \cdot \mathrm{s}$ at share rates of $10^{4} \mathrm{~s}^{-1}$ ).

Wakisaka et al. ${ }^{(8)}$ used a linear relationship between the flow rate and the ratio of the motor power to the square of the motor rotational speed. Although they used the hematocrit to compensate for changes in viscosity, this required invasive sampling of the blood. Granegger et al. ${ }^{(6)}$ derived cubic equations with regard to the current and rotational speed of the motor to estimate the flow rate. One difficulty of using this method is that determining the parameters in the equation is complicated because it contains 19 coefficients. Tsukiya et al. ${ }^{(9)}$ also estimated the flow rate from the current and rotational speed of the motor. Moreover, these signals were also used to estimate blood viscosity at a flow rate of zero by clamping an outlet port. All the methods described above use the current or power of the motor. However, the estimated flow rate and viscosity are susceptible to errors due to losses, such as copper loss and steel loss, which occur in the current and power of the motor. If the directly measured motor torque 
of an RBP is used for the estimation, the effect of the motor loss can be eliminated, and estimates with higher accuracy can be achieved even with a simple equation.

The purpose of this study is to realize a simple method for making highly accurate estimates of the flow rate in an RBP by using the directly measured motor torque, instead of using the motor power and current. The viscosity of the working fluid was estimated in real time using the motor torque at a flow rate of zero, and this was used to compensate the flow rate estimates. The accuracy of the proposed method was evaluated experimentally using glycerol-water mixtures with several viscosities. Although we used a previously developed centrifugal blood pump with a magnetic bearing, ${ }^{(10,11)}$ as shown in Fig. 1, the proposed method can also be applied to conventional RBPs with a contact bearing supporting the impeller.

\section{Materials and Methods}

\subsection{Principle of estimating the flow rate}

Equations for estimating flow rate by using the torque and rotational speed of the motor are proposed in this section. Since the specific gravity of blood, which is 1.05 , is almost constant, the only parameter we need to consider is the viscosity of the blood. Although blood behaves as a non-Newtonian fluid at shear rates below $100 \mathrm{~s}^{-1}$, (12) that in RBPs is higher than $10^{4} \mathrm{~s}^{-1}$, and we treat blood as a Newtonian fluid.

In rotary pumps, the following relationships among the motor torque $T$, the rotational speed of the motor $n$, and the flow rate $Q$ are obtained from dimensional analysis: ${ }^{(13)}$

$$
T \propto n^{2},
$$

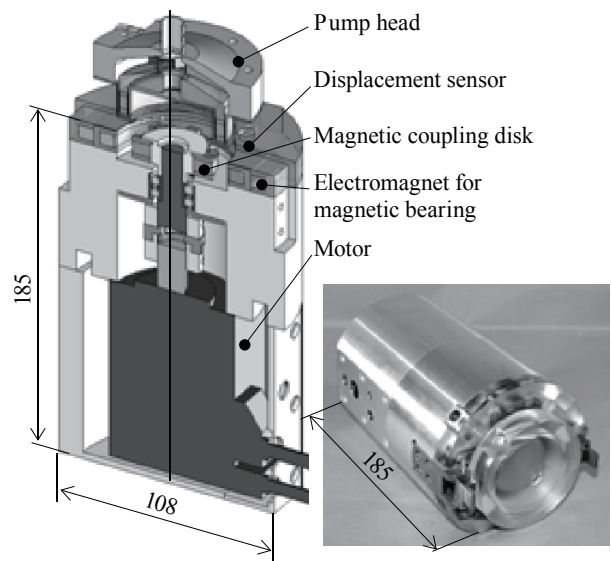

(a)

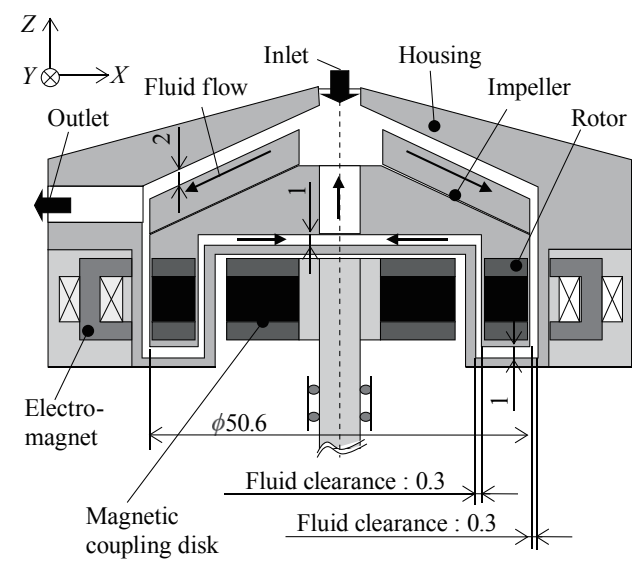

(b)

Fig. 1. Previously developed centrifugal blood pump with a magnetic bearing. (a) Configuration and photograph. (b) Cross-sectional view of the pump head. 


$$
Q \propto n .
$$

Therefore, $T$ is proportional to $n Q$. In practice, the motor torque $T$ consists of not only $n Q$ but also the torque due to hydrodynamic loss, $T_{L_{\text {hydro }}}$, and the torque due to mechanical loss, $T_{L_{\text {mech }}}$, such as the bearing loss. Therefore, the relationship between $T$ and $n Q$ is described as

$$
T=a n Q+T_{L_{\text {hydro }}}(n, \mu)+T_{L_{\text {mech }}}(n),
$$

where $a$ is a constant. $T_{L_{\text {hydr }}}$ is a function of the rotational speed of the motor, $n$, and the viscosity of the working fluid, $\mu$, while $T_{L_{\text {mech }}}$ is a function of the rotational speed only. In this study, $T_{L_{\text {mech }}}$ is directly measured and subtracted from eq. (3) in advance. Hence, we need to consider only an $Q$ and $T_{L_{\text {hydro }}}$ for the estimation. By dividing eq. (3) by an, we obtain the following equation for the flow rate:

$$
Q=a^{\prime} \frac{T}{n}+L_{\mathrm{hydro}}(n, \mu)
$$

where $a^{\prime}$ is the reciprocal of $a$. $L_{\text {hydro }}(n, \mu)$ is equal to $T_{L_{\text {hydro }}}(n, \mu)$ divided by $-a n$. The function $L_{\text {hydro }}(n, \mu)$ is obtained experimentally by varying the rotational speed $n$ and viscosity $\mu$. This is described in the following section.

\subsection{Principle of real-time estimates of the viscosity}

In order to compensate for viscosity in eq. (4), real-time estimates of the viscosity are used. The principle used for this is the same as that used in a rotational viscometer. To estimate the viscosity, the flow rate is set to zero by clamping the outlet port, and the rotational speed of the motor is set to a particular value, $n_{0}$. For example, $n_{0}$ is set to $1500 \mathrm{rpm}$ in this study. From eq. (3), the motor torque is given by the following equation with $Q=0$ :

$$
T=T_{L_{\text {hydro }}}\left(n_{0}, \mu\right)+T_{L_{\text {mech }}}\left(n_{0}\right) .
$$

Since the lateral fluid clearance of $0.3 \mathrm{~mm}$ shown in Fig. 1(b) is small, viscous shear stress generated on the impeller in this region is dominant. The Reynolds number in this region at $1500 \mathrm{rpm}$ with a blood viscosity of $3 \mathrm{mPa} \cdot \mathrm{s}$ is less than 400 . Therefore, the flow velocity in the fluid clearance region is expected to be similar to Couette flow, as shown in Fig. 2. Under this condition, $T_{L_{\text {hydro }}}$ is described by

$$
T_{L_{\text {hydro }}}\left(n_{0}, \mu\right)=S \cdot \tau \cdot r=S \cdot \mu \frac{r \omega_{0}}{d} \cdot r=S \cdot \mu \frac{2 \pi r^{2} n_{0}}{60 d}
$$

where $S$ is the lateral surface area of the impeller in the fluid clearance region, $\tau$ is the viscous shear stress, $r$ and $\omega_{0}$ are the radius and angular velocity of the impeller, respectively, and $d$ is the fluid clearance. From eqs. (5) and (6), the following 
relationship can be derived:

$$
\begin{aligned}
T-T_{\text {water }} & =\left\{T_{L_{\text {hydro }}}\left(n_{0}, \mu\right)+T_{L_{\text {mech }}}\left(n_{0}\right)\right\}-\left\{T_{L_{\text {hydro }}}\left(n_{0}, \mu_{\text {water }}\right)+T_{L_{\text {mech }}}\left(n_{0}\right)\right\} \\
& =K\left(\mu-\mu_{\text {water }}\right),
\end{aligned}
$$

where $T_{\text {water }}$ is the motor torque given by eq. (5) with water as the working fluid at a flow rate of zero, $\mu_{\text {water }}$ is the viscosity of water and $K$ is a constant, which is a function of $S, r$, $n_{0}$, and $d$. By knowing $T_{\text {water }}, K$, and $\mu_{\text {water }}$ in advance, the viscosity of the working fluid, $\mu$, can be estimated from eq. (7) in real time by measuring the motor torque $T$.

\subsection{Devices and the mock circulatory loop}

A magnetically levitated centrifugal blood pump developed by our group ${ }^{(10,11)}$ the configuration and a photograph of which are shown in Fig. 1, was used for the experiment. The pump consists of a motor, a magnetic coupling disk for torque transmission, an electromagnet for magnetic levitation of the 50.6-mm-diameter impeller and a pump head.

Figure 3 shows a schematic view of the measurement system. The torque meter is located between the magnetic coupling disk and the motor. The pump is connected to a mock circulatory loop consisting of a screw clamp, which simulates the fluid resistance of the cardiovascular system, a reservoir and an ultrasonic flow meter (HT-320, Transonic System Inc., USA, bandwidth: $10 \mathrm{~Hz}$ ), which is used to measure the reference flow rate $Q_{\text {ref. }}$ In order to control the temperature of the working fluid at $37^{\circ} \mathrm{C}$, the reservoir was put into a water bath with a thermoregulator.

If coagulation or hemolysis of the blood occurs, the measurement conditions such as the viscosity and torque loss can change unexpectedly. Therefore, in order to eliminate
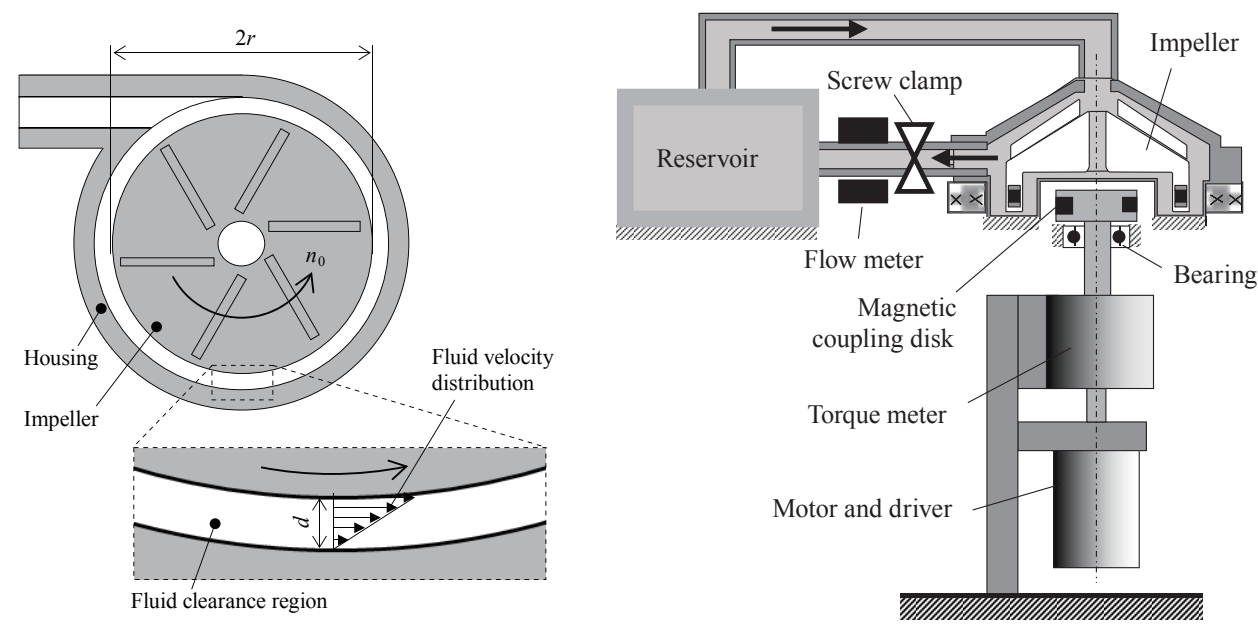

Fig. 2 (left). Top view of the centrifugal blood pump.

Fig. 3 (right). Schematic view of the measurement system. 
these uncertainties as much as possible, we used a mixture of glycerol and water, which is a Newtonian fluid, for the experiments. Working fluids with four different viscosities ranging from 0.75 to $3.29 \mathrm{mPa} \cdot \mathrm{s}$ were made by changing the glycerol concentration, as shown in Table 1. The fluid with $50 \mathrm{wt} \%$ glycerol with a viscosity of $3.29 \mathrm{mPa} \cdot \mathrm{s}$ simulates normal human blood. ${ }^{(14)}$ The other three fluids simulate blood with lower viscosity due to hemodilution. The reference viscosity of the working fluid was measured using a vibrational viscometer (SV-10, A\&D Co., Ltd., Japan, range: 0.3 to $10000 \mathrm{mPa} \cdot \mathrm{s}$, repeatability: $1 \%$, accuracy: $\pm 3 \%$ ).

In order to evaluate the effect of the accuracy of the torque measurement on the flow rate estimation, the motor torque was measured using both the torque monitor function of a commercially available motor driver (NX620AA, Oriental Motor Co., Ltd., Japan) and the torque meter (MD-503C, ONO SOKKI Co., Ltd., Japan, range: 0 to $500 \mathrm{mNm}$, resolution: $0.1 \mathrm{mNm}$ ). The torque measured using the motor driver, $T_{\text {driver, }}$ is obtained from the power consumption of the motor. Because of the copper and steel losses in the motor, $T_{\text {driver }}$ is considered to be less accurate than the measurement made with the torque meter, $T_{\text {meter }}$ The mechanical torque loss $T_{L_{\text {mech }}}$ in eq. (3) was measured by rotating the magnetic coupling disk without the pump head attached, and this was subtracted from $T_{\text {driver }}$ and $T_{\text {meter }}$ in advance.

\subsection{Determination of the equation for estimating the flow rate and viscosity}

By varying the rotational speed, the fluid resistance and the viscosity of the working fluid, we were able to ascertain the relationship between the flow rate $Q$ and the torque divided by rotational speed, $T / n$. From the measurements, the constant $a^{\prime}$ and the function $L_{\text {hydro }}(n, \mu)$ in eq. (4) were determined. The rotational speed of the motor was varied from 1000 to $3000 \mathrm{rpm}$ in increments of $250 \mathrm{rpm}$. The four different working fluids shown in Table 1 were used.

In addition, the relationship between $T-T_{\text {water }}$ and $\mu-\mu_{\text {water }}$ was also measured at a rotational speed $n_{0}$ of $1500 \mathrm{rpm}$. The working fluids were those in Table 1 and the outlet port was clamped. From the results, the constant $K$ in eq. (7) was determined.

\subsection{Evaluation of the accuracy of the estimated flow rate}

Using eq. (4), we evaluated the accuracy of the estimated flow rate. Firstly, flow rates were estimated using both the motor torque obtained from the motor driver, $T_{\text {driver }}$, and that measured directly using the torque meter, $T_{\text {meter, }}$ and their accuracies were compared. In this measurement, the working fluid was just water. Hence, the viscosity $\mu$ in eq. (4) was fixed to $0.75 \mathrm{mPa} \cdot \mathrm{s}$ and real-time estimates of viscosity were not used. The flow

Table 1

Viscosities of working fluids at $37^{\circ} \mathrm{C}$.

\begin{tabular}{ll}
\hline Water & $0.75 \mathrm{mPa} \cdot \mathrm{s}$ \\
$30 \mathrm{wt} \%$ glycerol & $1.61 \mathrm{mPa} \cdot \mathrm{s}$ \\
$40 \mathrm{wt} \%$ glycerol & $2.22 \mathrm{mPa} \cdot \mathrm{s}$ \\
$50 \mathrm{wt} \%$ glycerol & $3.29 \mathrm{mPa} \cdot \mathrm{s}$ \\
\hline
\end{tabular}


rate was varied from 0 to $10 \mathrm{~L} / \mathrm{min}$, which adequately covers the circulation support needed for failing hearts.

Secondly, flow rates were estimated using the fluids with 30,40 , and $50 \mathrm{wt} \%$ glycerol shown in Table 1. In this experiment, real-time measurements were used for estimating viscosity, and the effectiveness of the viscosity compensation was evaluated.

\section{Results}

\subsection{Determination of the equation for estimating the flow rate}

Figure 4 shows the measured relationships between the reference flow rate $Q_{\text {ref }}$ and the motor torque measured using the torque meter divided by the rotational speed, $T_{\text {meter }} / n$, at various constant rotational speeds in water, and, as expected from eq. (4), the relationships obtained are linear. Figure 5 shows the intercepts of these linear relationships, i.e., where $T_{\text {meter }} / n=0$. At each intercept, $L_{\text {hydro }}(n, \mu)=Q_{\text {ref }}$, and $L_{\text {hydro }}(n, \mu)$ is found to be linearly proportional to the rotational speed. Therefore, the function $L_{\text {hydro }}(n, \mu)$ can be described by

$$
L_{\text {hydro }}(n, \mu)=b(\mu) n+c(\mu) \text {, }
$$

where $b(\mu)$ and $c(\mu)$ are functions of the viscosity.

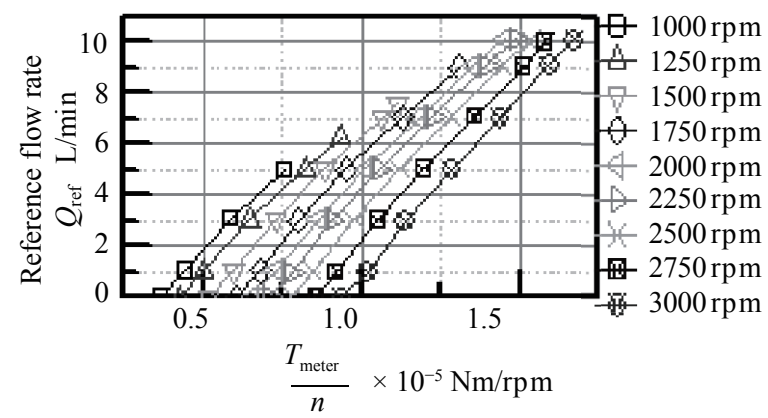

Fig. 4. Relationship between $Q_{\text {ref }}$ and $T_{\text {meter }} / n$ in water.

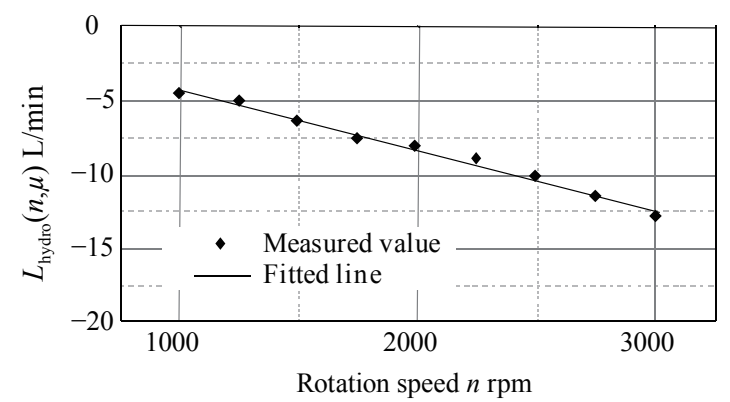

Fig. 5. Relationship between $L_{\mathrm{hydro}}(n, \mu)$ and rotational speed in water. 
Using the other three working fluids listed in Table 1, the same measurements were conducted, and $a^{\prime}, b(\mu)$, and $c(\mu)$ were obtained for each fluid. Figure 6 shows the relationship between $b(\mu)$ and the viscosity $\mu$. In order to derive a simple equation for the flow rate estimation, $b(\mu)$ is linearized as in eq. (9):

$$
b(\mu)=b_{1} \mu+b_{0},
$$

where $b_{1}$ and $b_{0}$ are constants. In the same way, $c(\mu)$, shown in Fig. 7, is also linearized:

$$
c(\mu)=c_{1} \mu+c_{0}
$$

where $c_{1}$ and $c_{0}$ are constants. Therefore, from eqs. (4), (8), (9), and (10), the equation for estimating flow rate is finally derived as

$$
Q=a^{\prime} \frac{T}{n}+\left(b_{1} \mu+b_{0}\right) n+c_{1} \mu+c_{0} .
$$

Table 2 shows the experimentally determined constants used in eq. (11) for estimating flow rate. Note that $a^{\prime}$ is the average value obtained from all four fluids.
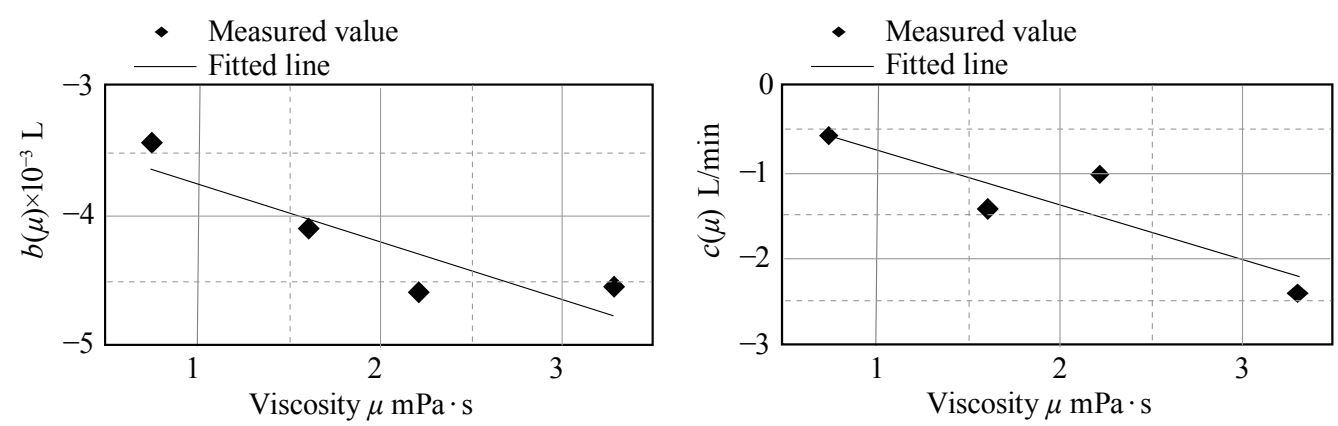

Fig. 6 (left). Relationship between $b(\mu)$ and viscosity.

Fig. 7 (right). Relationship between $c(\mu)$ and viscosity.

Table 2

Experimentally determined parameters for estimating flow rate.

\begin{tabular}{lc}
\hline$a^{\prime}[\mathrm{L} \cdot \mathrm{rpm} /(\mathrm{Nm} \cdot \mathrm{min})]$ & $1.31 \times 10^{6}$ \\
$b_{0}[\mathrm{~L}]$ & $-4.45 \times 10^{-5}$ \\
$b_{1}[\mathrm{~L} /(\mathrm{mPa} \cdot \mathrm{s})]$ & $-3.30 \times 10^{-3}$ \\
$c_{0}[\mathrm{~L} / \mathrm{min}]$ & -0.65 \\
$c_{1}[\mathrm{~L} /(\mathrm{mPa} \cdot \mathrm{s} \cdot \mathrm{min})]$ & $-9.45 \times 10^{-2}$ \\
\hline
\end{tabular}




\subsection{Determination of the equation for estimating viscosity}

Figure 8 shows the relationship between $T-T_{\text {water }}$ and $\mu-\mu_{\text {water }}$ at a rotational speed $n_{0}$ of $1500 \mathrm{rpm}$. The result was linearized and the proportionality coefficient $K$ was determined to be $1.70 \mathrm{Nm} /(\mathrm{Pa} \cdot \mathrm{s})$. In this measurement, $T_{\text {water, }}$, which was obtained in advance using the torque meter at the same rotational speed, was $0.012 \mathrm{Nm}$ and $\mu_{\text {water }}$ was $0.75 \mathrm{mPa} \cdot \mathrm{s}$.

Using eq. (7), real-time viscosity estimates were conducted and compared with the reference viscosities measured using the viscometer, as shown in Fig. 9. An estimation error of less than $0.16 \mathrm{mPa} \cdot \mathrm{s}$ was achieved in the range from 0.75 to $3.29 \mathrm{mPa} \cdot \mathrm{s}$.

\subsection{Effect of the accuracy of the torque measurements on the flow rate estimation}

Figure 10 shows a comparison between the reference flow rates measured using the ultrasonic flow meter and the estimated flow rates. The torque obtained from the motor driver, $T_{\text {driver, }}$, was used to estimate the flow rates for Fig. 10(a), while the torque measured directly using the torque meter, $T_{\text {meter, }}$ was used for Fig. 10(b). The differences between the reference flow rates and the estimated ones using $T_{\text {meter }}$ are less than $0.5 \mathrm{~L} / \mathrm{min}$ in the range from 0 to $10 \mathrm{~L} / \mathrm{min}$, which is $50 \%$ smaller than the equivalent value using $T_{\text {driver }}$.

\subsection{Flow rate estimation with viscosity compensation}

Figure 11 shows a comparison between the estimated flow rate and the reference flow rate for the 30, 40, and $50 \mathrm{wt} \%$ glycerol-water solutions. Figure 11(a) shows the results without viscosity compensation. In this measurement, the viscosity $\mu$ in eq. (4) was fixed to the viscosity of the $40 \mathrm{wt} \%$ solution, i.e., $2.22 \mathrm{mPa} \cdot \mathrm{s}$, and the estimation error was less than $2.0 \mathrm{~L} / \mathrm{min}$ in the range from 0 to $10 \mathrm{~L} / \mathrm{min}$. Figure 11(b) shows the results with viscosity compensation using the real-time viscosity estimation for each working fluid. The estimation error has improved to less than $0.77 \mathrm{~L} / \mathrm{min}$.
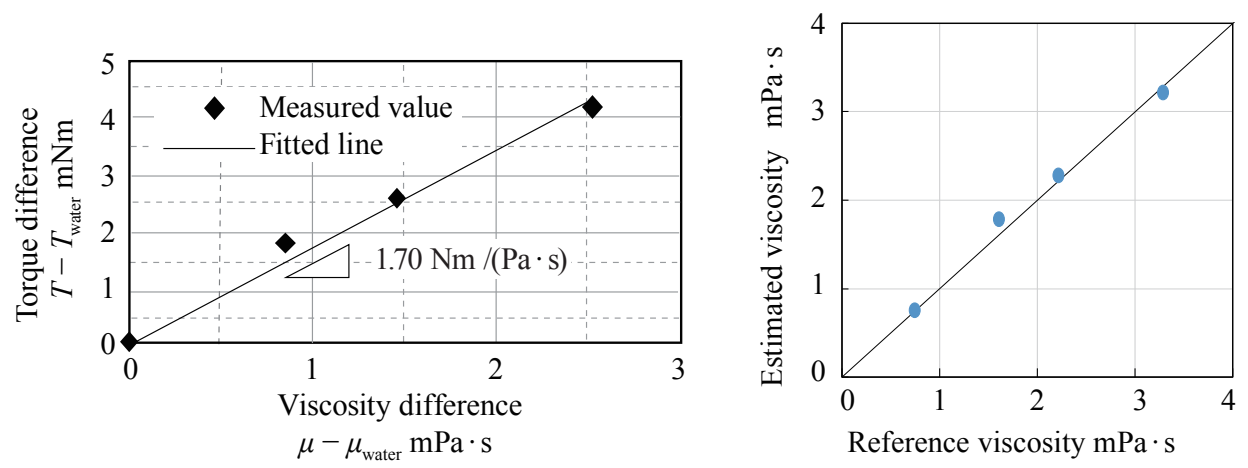

Fig. 8 (left). Relationship between $T-T_{\text {water }}$ and $\mu-\mu_{\text {water }}$ at a rotational speed of $1500 \mathrm{rpm}$. Fig. 9 (right). (Color online) Relationship between the reference and estimated viscosities. 


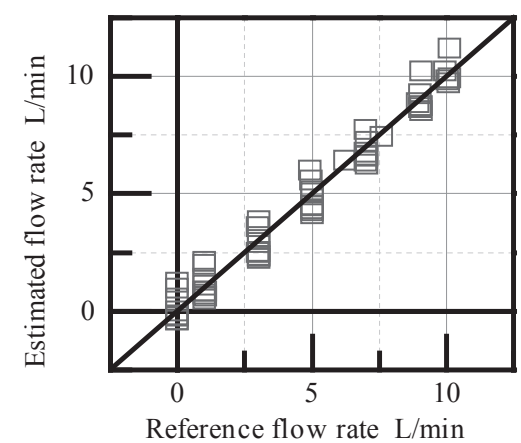

(a)

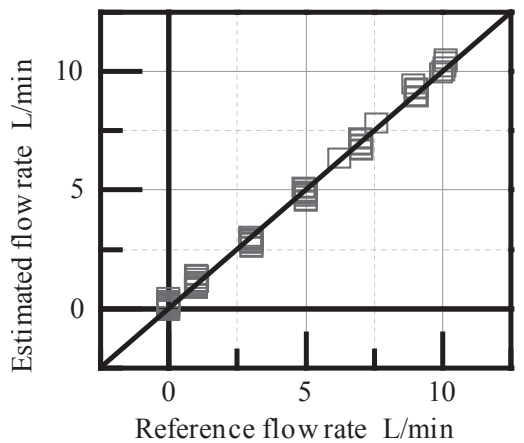

(b)

Fig. 10. Comparison between the reference and estimated flow rates with water as the working fluid at $37^{\circ} \mathrm{C}$. (a) Using the motor driver torque $T_{\text {driver }}$ (b) Using the output of the torque meter $T_{\text {meter }}$

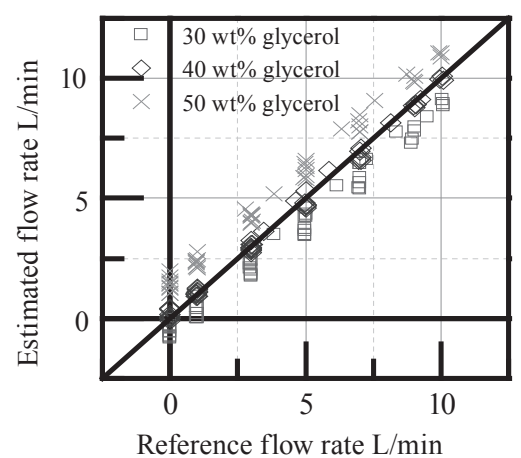

(a)

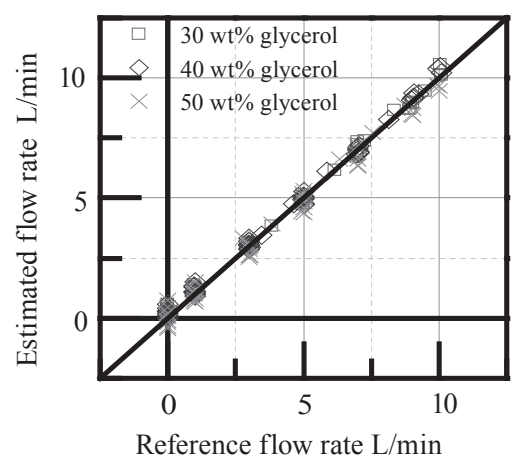

(b)

Fig. 11. Comparison between reference and estimated flow rates. (a) Without viscosity compensation. (b) With viscosity compensation.

\section{Discussion}

In the measurement results shown in Fig. 10, the estimation accuracy of the flow rate using $T_{\text {meter }}$ is less than half that using $T_{\text {driver }}$. Since $T_{\text {driver }}$ is calculated from the motor power, it has a measurement error affected by motor losses, such as copper loss and steel loss. The experimental results demonstrate that more accurate torque measurements would improve the accuracy of the flow rate estimation. Owing to the accurate torque measurements, the error in estimating the flow rate, which is $0.77 \mathrm{~L} / \mathrm{min}$ in the range from 0 to $10 \mathrm{~L} / \mathrm{min}$, is comparable to that found by other researchers ${ }^{(4,6,8,9)}$ even though the equation for estimating the flow rate is much simpler. Therefore, the proposed estimation method has an advantage in that it requires less calibration procedures. 
The proposed method can be applied to extracorporeal circulation support, in which an RBP is placed outside the patient's body. However, for use in implantable RBPs, it is necessary to make the torque measurement mechanism much smaller. Furthermore, the real-time viscosity estimations used in this study require the flow rate to be set temporarily to zero. This is undesirable for an implantable RBP because the additional clamping mechanism would need to be placed inside the body. Instead, we have developed a novel method for estimating viscosity by vibrating the impeller using an electromagnet for the magnetic bearing. ${ }^{(15)}$ In this method, it is not necessary to set the flow rate to zero, and thus no additional mechanisms are required. In our next work, the proposed flow rate estimation method described in this study and the viscosity estimation method described in ref. 15 will be combined and evaluated with the objective of developing this for use in implantable RBPs.

\section{Conclusions}

Methods of estimating the flow rate and viscosity in a RBP using accurate measurements of the torque and rotational speed of the motor were developed. The estimation error achieved was less than $0.77 \mathrm{~L} / \mathrm{min}$ in the range from 0 to $10 \mathrm{~L} / \mathrm{min}$. By clamping the outlet port, the viscosity of the working fluid could be estimated from the motor torque. The error in estimating the viscosity was less than $0.16 \mathrm{mPa} \cdot \mathrm{s}$ in the range from 0.75 to $3.29 \mathrm{mPa} \cdot \mathrm{s}$, which is sufficient for viscosity compensation. Even though the proposed method for estimating the flow rate is described using a simple equation, the accuracy of the method is comparable to those reported in previous research owing to the highly accurate torque measurements and real-time viscosity compensation. This advantage decreases the number of calibration procedures required for estimating the flow rate.

Future work includes evaluating the proposed method using blood. Furthermore, we plan to combine this method with a method for estimating viscosity that does not require clamping of the outlet port so that it can be applied to implantable RBPs.

\section{Acknowledgements}

This study was partly supported by JSPS KAKENHI Grant Number 24360059.

\section{References}

1 World Health Organization (WHO): Causes of death by age, sex and region, 2000-2012, http://www.who.int/healthinfo/global_burden_disease/en/ (accessed on January 2015).

2 F. D. Pagani: Semin. Thorac. Cardiovasc. Surg. 20 (2008) 255.

3 H. Hoshi, T. Shinshi and S. Takatani: Artif. Organs 30 (2006) 324.

4 C. D. Bertram: Physiol. Meas. 26 (2005) 99.

5 M. Arakawa, T. Nishimura, Y. Takewa, A. Umeki, M. Ando, H. Adachi and E. Tatsumi: J. Thorac. Cardiovasc. Surg. 148 (2014) 698.

6 M. Granegger, M. Moscato, F. Casas, G. Wieselthaler and H. Schima: Artif. Organs 36 (2012) 691. 
7 K. Karkouti, W. S. Beattie, D. N. Wijeysundera, V. Rao, C. Chan, K. M. Dattilo, G. Djajani, J. Ivanov, J. Karski and T. E. David: J. Thorac. Cardiovasc. Surg. 129 (2005) 391.

8 Y. Wakisaka, Y. Okuzono, Y. Taenaka, K. Chikanari, T. Masuzawa, T. Nakatani, E. Tatsumi, T. Nishimura, Y. Takewa, T. Ohno and H. Takano: Artif. Organs 21 (1997) 651.

9 T. Tsukiya, T. Akamatsu, K. Nishimura, T. Yamada and T. Nakazeki: Artif. Organs 21 (1997) 396.

10 W. Hijikata, T. Shinshi, J. Asama, L. Li, H. Hoshi, S. Takatani and A. Shimokohbe: Artif. Organs 32 (2008) 531.

11 T. Someya, M. Kobayashi, S. Waguri, T. Ushiyama, E. Nagaoka, W. Hijikata, T. Shinshi, H. Arai and S. Takatani: Artif. Organs 33 (2009) 704.

12 S. Chien: Science 168 (1970) 977.

13 E. J. Finnemore and J. B. Franzini: Fluid Mechanics with Engineering Applications (McGraw-Hill Higher Education, Columbus, 2001).

14 O. K. Baskurt and H. J. Meiselman: Semin. Thromb. Hemost. 29 (2003) 435.

15 W. Hijikata, J. Rao, S. Abe, S. Takatani and T. Shinshi: Artif. Organs (in press). 